\title{
The Design of the Drill Bit \& Sample Acquisition Mechanism DBSAM
}

\author{
Moortaza Bhaiji*, Michel El-Saddi*, Naji El-Khouri*, \\ Howard Jones $^{* *}$, and Alexei Morozov* \\ *Department of Mechanical Engineering, McGill University, Montreal, Quebec, Canada \\ ${ }^{* *}$ MD Robotics, Brampton, Ontario, Canada \\ alexvit@cim.mcgill.ca
}

\begin{abstract}
Space scientists and technologists spend more and more efforts developing principles and technologies for Mars exploration. One of the most important tasks is to analyze subsurface Martian soil and rocks for traces of past or present life. The Mars Lander should possess a reliable and compact drilling and sample acquisition mechanism which can be stored on board the Lander and be assembled automatically during operation. An important part of this mechanism is a drill bit and sample acquisition mechanism (DBSAM). Its primary functions are going to be drilling deep into rock or regolith and sample acquisition. Samples are to be either of particulate or solid material and must be contained within the device until ejection. This article discusses and elaborates a design of the DBSAM.
\end{abstract}

\section{Introduction}

As curiosity grows and technology advances, scientists and engineers alike, are now more than ever involved in the search for extra-terrestrial life. The planet Mars has been the latest focus of many missions. The Red Planet's similarities to the Earth: its atmosphere, evidence of past water reservoirs, etc. promises past or present life.

Past successful missions like the Mars Exploration Rover have only been able to analyze surface rocks, soil and other debris. Due to surface processes, most organic traces tend to be erased. This requires us to dig deeper into the Martian surface in our search for life.

The DBSAM project is an integral part of a Drill System. Its primary functions are drilling deep into rock or regolith and sample acquisition as the name suggests. Samples are to be either of particulate or solid material. The DBSAM will be mounted at the tip of the drill and be driven via drill rods to cut through rock.

The DBSAM must be able to cut through regolith and rock and to acquire a sample. The DBSAM will be capable of acquiring samples that will be well contained while it is brought to the surface. The sample holder will have protective sheathing to preserve the integrity of the sample while being obtained and returning to the surface. On withdrawal from the bored hole, and upon command, the sample is to be ejected into a sample holder by the DBSAM.

The DBSAM will enable scientists to analyze subsurface soil and rocks, using the tools available on the Lander or drill system itself, for traces of past or present life. The DBSAM will be the next step towards finding extra-terrestrial life!

\section{Design of the DBSAM}

The DBSAM design (Fig.1) consists of 3 main components:

- The Outer Cylinder

- The Piston Mechanism

- The Cutting Mechanism

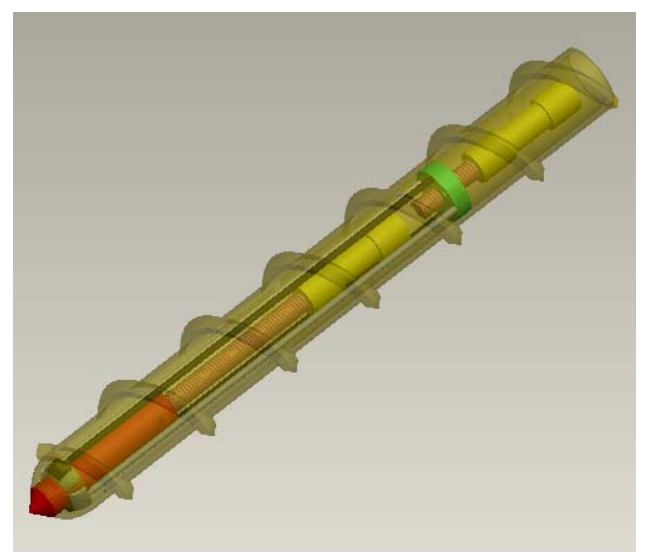

Fig.1. Fully Assembled DBSAM

\section{A. The Outer Cylinder}

The outer cylinder is the base structure of the whole design. It contains all the sub-mechanisms and provides the structural strength. 
This is a steel cylinder of $40 \mathrm{~mm}$ in diameter with a 19 $\mathrm{mm}$ hole bored through it. This acts as the outer sheathing and base structure for the whole mechanism. The cylinder has an auguring, $7.5 \mathrm{~mm}$ deep grooved into it, which functions as a waste removal tool during the drilling process.

The outer cylinder is capped by a similar steel piece on which the auger pattern is continued. The drilling end of this piece has $109^{\circ}$ angle and cutting edges to enable drilling. A $10 \mathrm{~mm}$ hole is bored through this piece. On the end, which attaches to the outer cylinder, there are four slots milled around the hole (Fig.2).

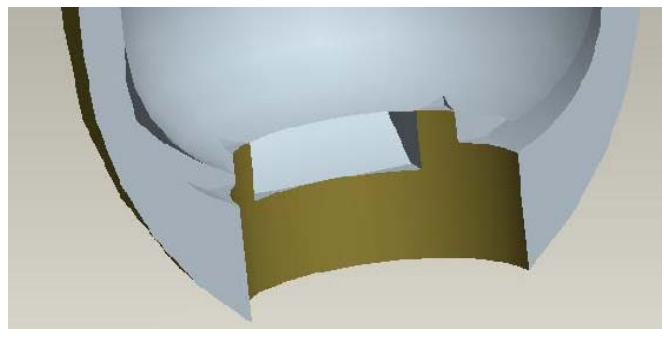

Fig.2. View of a slot

Within the outer cylinder two separate mechanisms are housed: the piston mechanism and the cutting mechanism.

\section{B. The Piston Mechanism}

The piston mechanism (Fig.3) is a sub unit of the DBSAM. It consists of the following components:

- Motor

- Screw

- Piston

- $\quad$ Slotted Cylinder

The piston is a $45-\mathrm{mm}$ long steel cylinder with a $109^{\circ}$ chamfer at one end. The piston has a $40 \mathrm{~mm}$ deep hole bored through its non-chamfered end. The first $15 \mathrm{~mm}$ of the hole are threaded. A pin is attached to one end of the piston to fit into the slot.

The slotted cylinder is a steel tube $90 \mathrm{~mm}$ in length. It has a $5 \mathrm{~mm}$ slot running for $55 \mathrm{~mm}$ along its length. There is also a slot-less length of about $40 \mathrm{~mm}$ where the sample will be stored. This length will be thermally insulated and provide protection to the sample.

A threaded screw of about $55 \mathrm{~mm}$ is attached to motor which in turn is attached to the top of the slotted cylinder. The free end of the screw connects to the piston. When the motor rotates, the piston rides up and down the length of the screw, along the slot.

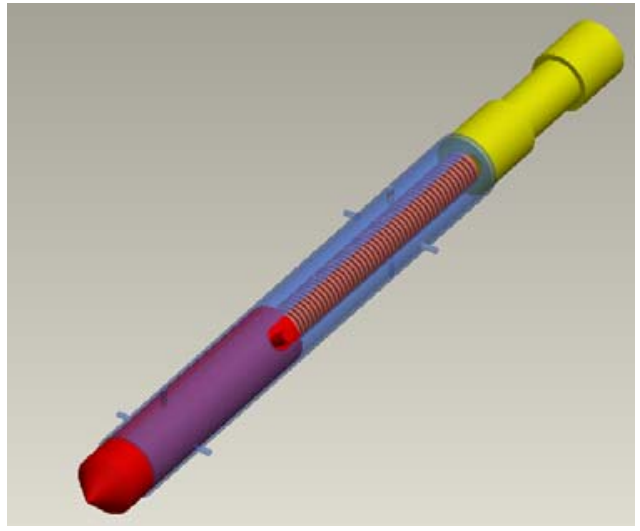

Fig.3. Piston Mechanism

\section{The Cutting Mechanism}

The cutting mechanism (Fig.4) is composed of the following parts:

- Motor

- Screw

- Steel Plate

- Cutting Rods

- Cutters

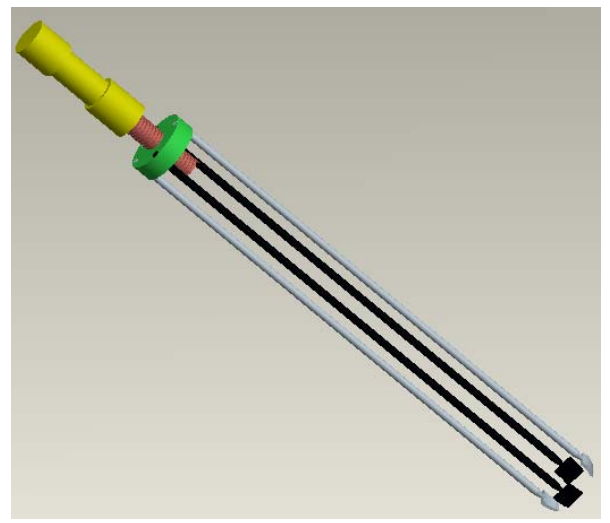

Fig.4. Cutting Mechanism

The cutting rods have $2 \mathrm{~mm}$ in diameter. Two rods are $139 \mathrm{~mm}$ in length and the other two $138 \mathrm{~mm}$ in length. The rods are tipped with cutters $8 \mathrm{~mm}$ in width and $4.87 \mathrm{~mm}$ in height. They have a similar profile to the slots in the cap and are expected to fit as male-female parts. Between the rod and the cutters a slightly elastic material, a ribbon, is to be used to allow bending of the rods to accommodate the cutting mode. 
The four rods are housed on a steel plate of $17 \mathrm{~mm}$ in diameter and $5 \mathrm{~mm}$ thick. A hole is bored and threaded through the middle to house a screw. The screw, similar to the screw in the piston mechanism, is attached to a motor. Four more holes of $2 \mathrm{~mm}$ are drilled into this piece to allow the cutting rods to be press fit into the plate.

\section{Finite Element Analysis}

The analysis of some key components of the DBSAM was done using Algor 14.2 (Fig.5). It was required that certain assumptions and engineering approximations be made.

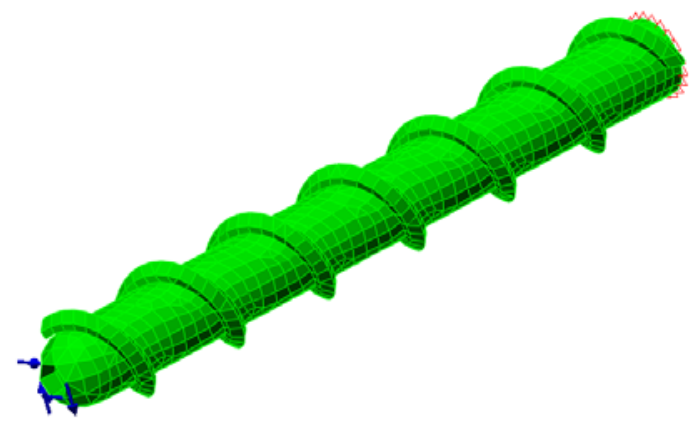

Fig.5. Boundary conditions on Outer Cylinder

Several of constraints were posed by the design problem itself and more by the Martian environment. Some important factors taken into account during the analysis include:

- Maximum thrust to be applied is $500 \mathrm{Nm}$.

- Maximum torque to be applied is $2.5 \mathrm{Nm}$.

- Martian temperature between $-10^{\circ} \mathrm{C}$ and $-130^{\circ} \mathrm{C}$.

- Martian rock properties - Hardness is 8 on Mohs Scale.

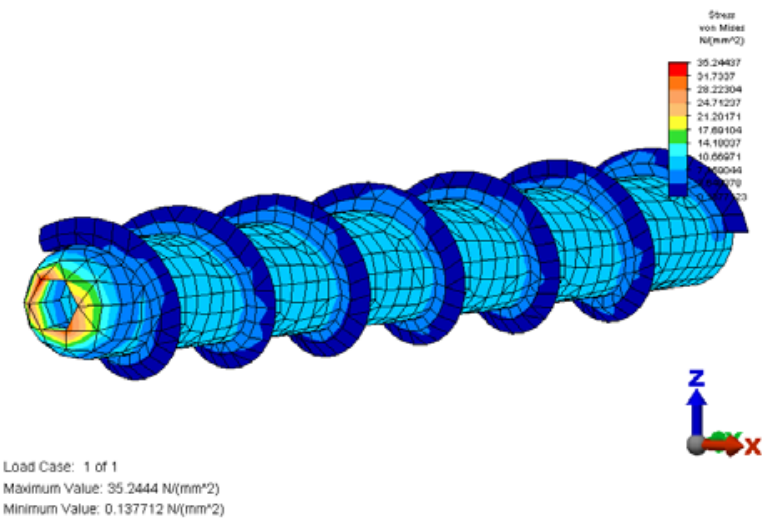

Fig.6. Stress analysis results
The outer cylinder was analyzed taking into account the thrust force and torque mentioned above as well as an average temperature of $-50^{\circ} \mathrm{C}$.

The results shown in Fig.6 indicate no signs of failure or points of weakness in the design. The model is depicted with an external auguring for ease of analysis and the results are valid for a grooved auguring.

The piston was analyzed for stresses and weakness under the following conditions (Fig.7):

- Thrust: $500 \mathrm{~N}$

- Torque: $2.5 \mathrm{Nm}$ around the $\mathrm{z}$-axis

- $\quad$ One end fixed

- $\quad$ Operating temperature set to $-50^{\circ} \mathrm{C}$

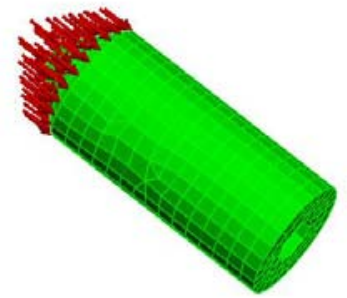

Fig.7. Conditions set on Piston

The piston is a key component in our design and will be subjected to large stresses during operation. The above conditions ensure that the piston will not be compromised during operation and as seen from Fig.8 the piston is relatively stress free.

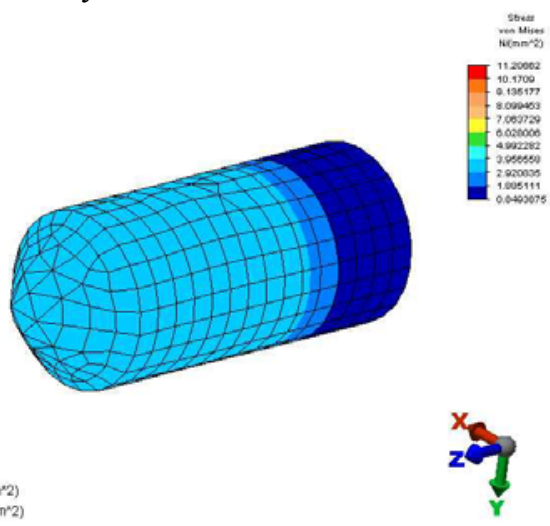

Fig.8. Piston analysis results

The Cutters and cutting rods were also analyzed. A cutting force of $15 \mathrm{~N}$ was applied at the tip of the cutters. This was determined after intensive research into rock drilling. The elastic material was set to be rigid as during operation it is to be supported by the 
slots. The analysis was carried out under similar environmental conditions as above and yielded positive results (Fig.9).

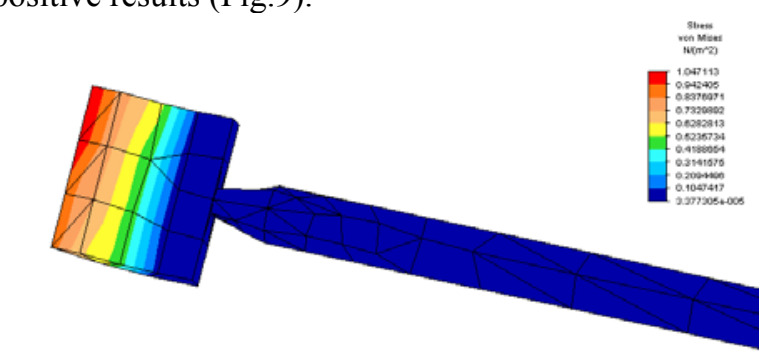

Load case 1 of 1 Macimum Value $1.047111 \mathrm{~N}\left(\mathrm{~m}^{\circ} 2\right)$

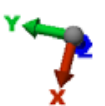

Fig.9. Stress concentration at cutting edge

The high stress concentration at the tip is due to material selection. For the analysis, a common steel was chosen but suitable diamond or carbide material as used for most drilling purposes will be used in the final product.

The screw was not analyzed as the proposed screw is an ACME screw and manufacturer properties have been deemed sufficient to withstand forces encountered.

\section{Functioning of the DBSAM}

The DBSAM when fully assembled is designed to have an outer diameter of $20 \mathrm{~mm}$ excluding the auguring and a maximum length of $200 \mathrm{~mm}$ (Fig.1). It is also designed to use a maximum of eight conductors. Currently the design does not include thermisters and pressure sensors which have been accounted for in the eight conductors. Redundancy will increase the number of conductors.

The functions of the three components of the DBSAM are outlined below.

\section{A. Outer Cylinder}

Ideally manufacturing the outer cylinder and the cap as one would have been an appropriate scenario but manufacturing complications has led to two different pieces.

The cylinder has an auguring carved out which acts as a waste removal tool during the drilling process. The auguring also has a cutting edge to assist in the drilling process.

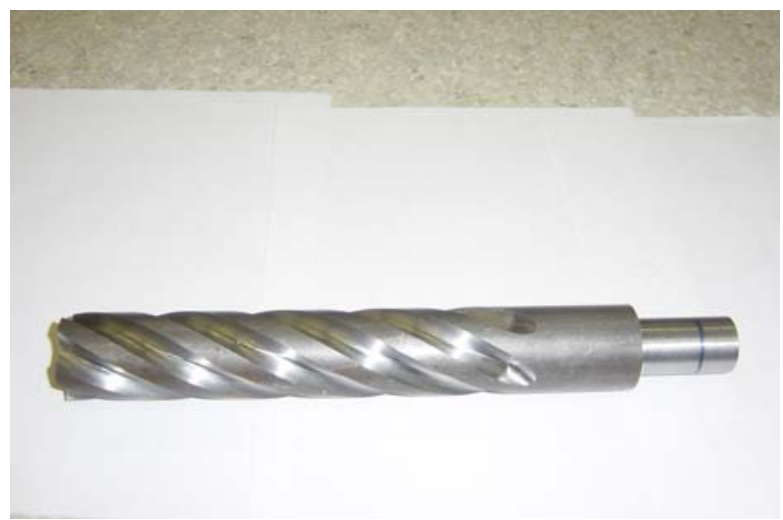

Fig.10. Outer Cylinder with auguring

The cap which is part of the outer cylinder contains a $10 \mathrm{~mm}$ hole through which the sample is to be acquired. The edges of this hole have cutting edges to assist the sampling process. The slots in the cap mentioned above are to contain and guide the cutters. They have been designed offset to prevent the cutters from colliding and jamming during the cutting process.

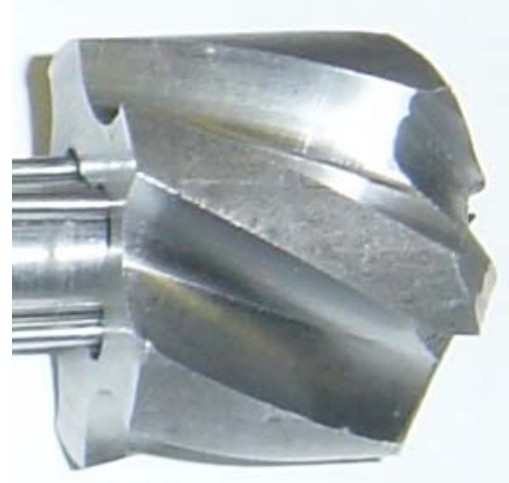

Fig.11. Cap with cutting rods in slots

The $109^{\circ}$ angle of the cap is the standard drill bit angle. The cap will also have several drilling tips to minimize the load on the piston.

\section{B. The Piston Mechanism}

The piston mechanism is composed of four key components, including the piston, slotted cylinder, screw and motor.

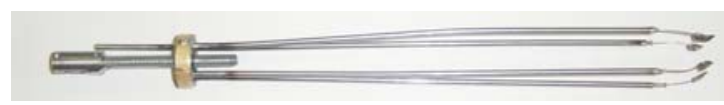

Fig.12. Cutting Mechanism without motor 
The motor is used to turn the screw which in turn controls the retraction and extension of the piston cylinder. When the piston is fully retracted it is in "sampling mode", i.e. any further drilling action will cause a core to be accumulated within the cavity formed by the retraction. This core will be supported and sheathed by the cylinder to protect it from contamination. After coring and sampling the sample is ejected by the extending the piston back to its original position, drilling configuration, thus pushing the sample out. As seen in the drawing the piston has a pin attached to its base. This is used in conjunction with the slot in the screw cylinder, to guide the piston up and down the screw and prevent rotation during drilling, retraction and extension. The slotted cylinder is also going to be used to house the motor and the screw. The screw cylinder is attached to the cap of the mechanism by welding.

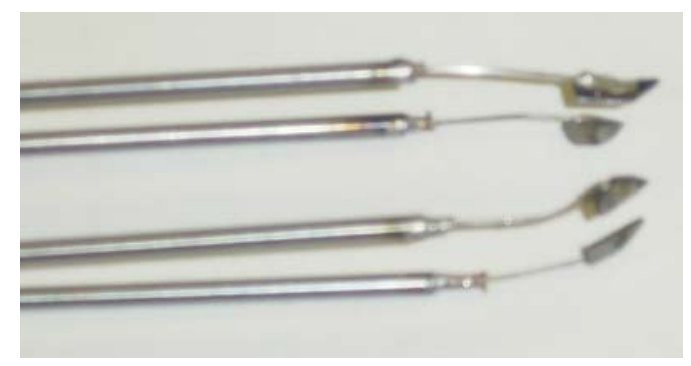

Fig.13. Cutters close-up

\section{The Cutting Mechanism}

The cuttings rods of the Cutting Mechanism are held between the steel plate and the slots in the cap. The steel plate has four holes into which the cutting rods are fixed. From here they extend down to the slots where they fit snugly to prevent rotation of the steel plate. The cutters are attached to the cutting rods via a flexible ribbon and when activated close to completely cover the cavity left by the retraction of the piston.

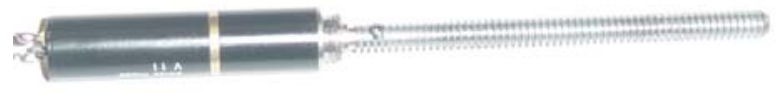

Fig.14. Maxxon RE-10 motor and screw of Piston Mechanism

The cutters are activated by the motor that turns the ACME screw, which in turn causes the steel plate to move up or down according to the function required. In its original position the cutters allow the piston through and therefore can be considered in drilling configuration. After sampling has occurred, i.e. the piston has been retracted and a sample cored, the cutters are pushed down into the slots by the activation of the motor. The ribbons bend and the cutters emerge slightly from the slots to begin the cutting process. As the sample is scored the cutters are fed towards the center axis of the whole device. Due to the offset, two cutters meet above the other two cutters. When this happens the sample has been safely stored in the inside cylinder and the only exit point is fully sealed by the cutters. On ejection of the sample the cutters are withdrawn to their original configuration i.e. in the slots.

\section{The DBSAM Prototype}

A preliminary prototype (Fig.10) was built as a proof of concept and was manufactured to drill into a soft material such as wood or foam.

Steel was used as the primary material for the prototype. The ribbons were replaced by guitar strings to provide flexibility and the cutters were made out of simple saw teeth. The Figs.11-17 show the details of the prototype and the final assembly.

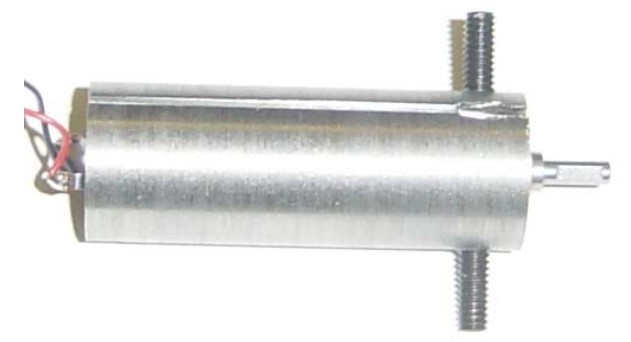

Fig.15. Maxxon RE-13 motor in holder for the Cutting Mechanism

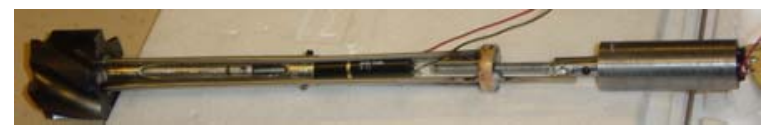

Fig.16. Cap, Piston and Cutting Mechanism assembly

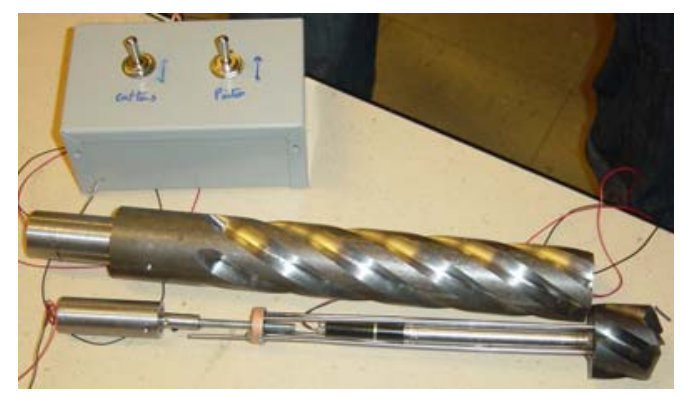

Fig.17. All components prior to complete assembly 
The screw that was used for the prototype was $3 / 4 ", 10$ 24 UNC threaded steel rod. We also used two Maxxon motors a 10mm, 64:1 geared RE-10 and a $13 \mathrm{~mm}, 67: 1$ geared RE-13 motor for the piston and cutting mechanism respectively. These motors provided sufficient torque and are well within the power constraint of $10 \mathrm{~W}$ as specified by the design constraints.

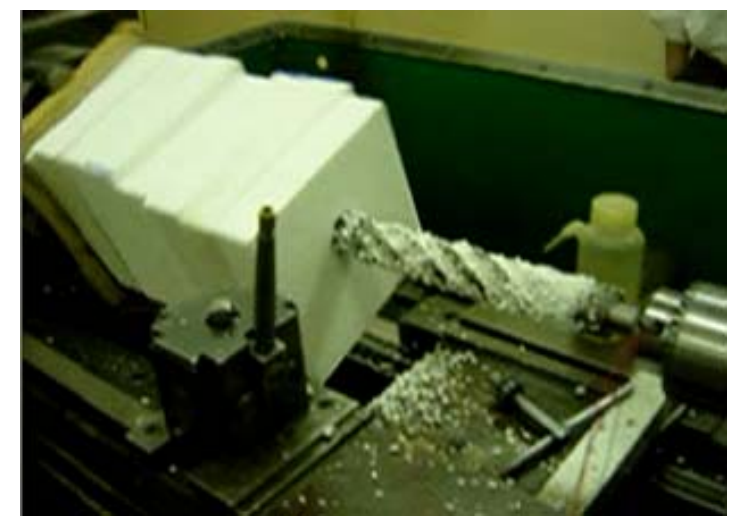

Fig.18. DBSAM drilling into foam

\section{Testing the DBSAM Prototype}

The prototype was done by mounting the DBSAM on a lathe and rotating the material using the lathe (Fig.18). This simulated drilling action but the DBSAM was not rotated. The material used for the proof of concept test was foam. Several tests were performed and samples taken (Figs.19, 20). At every successive the DBSAM was made to drill deeper and acquire larger samples.

\section{Results}

The drilling tests were successful and conclusive.

The tests proved that the initial design concept is feasible and can be manufactured to perform on the Martian environment.

\section{Conclusions}

The Drill Bit Sample and Acquisition Mechanism is more than a mechanical/electrical device. It might be the device that will establish a breakthrough in the search for life on Mars. It is a device that is able to drill into the Martian surface, sample and eject a core all in a simple system. Initially, several concepts were studied and analyzed in detail for their performance. The final concept has been a result of a long and organized study.

One must understand the goal and necessities of the actual device. The prototype was built to complete its tasks on soft material (wood or foam), the material used is definitely not appropriate for the Martian environment, considering the differences in pressure, temperature, hardness of soil particles. However, its dimensions are unchanged and its whole process is exactly what will be expected if the prototype was to be produced.

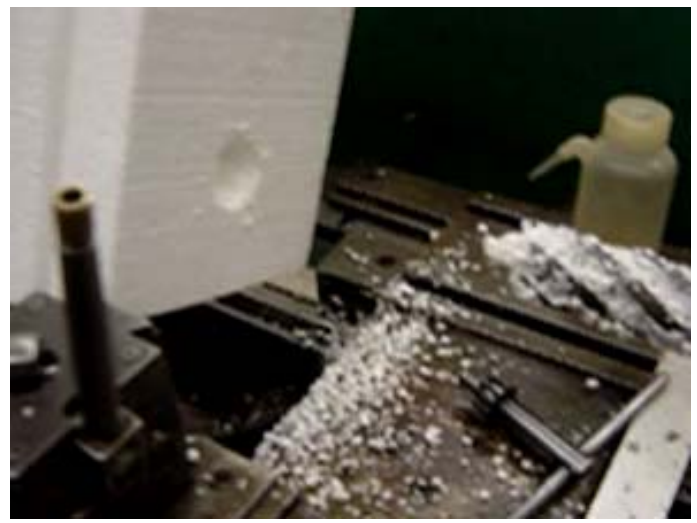

Fig.19. DBSAM with sample stored

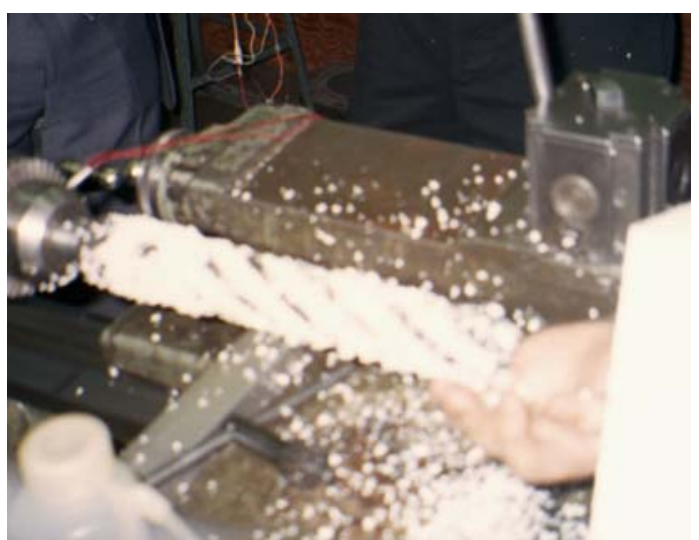

Fig.20. DBSAM ejecting samples onto hand

Some recommendations are outlined below:

- The properties and conditions of the Mars environment must be studied in detail to determine the suitable modifications that have to be done on the prototype.

- Material selection must be reconsidered and space rated materials suitable for the design must be used. 
- The control mechanism should be improved and temperature and pressure sensors included to ensure integrity of the sample.

\section{Acknowledgements}

We would like to express our appreciation to professor Jorge Angeles for his support during the work on the project, and to Machine tool lab experts of McGill University for their valuable help in building and testing of the DBSAM prototype.

\section{REFERENCES}

[1]. Boresi, Arthur P., Schmidt, Richard J., 2003, Advanced mechanics of materials, New York: John Wiley \& Sons.

[2] Ketchum, Milo S., 1912, The design of mine structures, New York: McGraw-Hill.

[3] Shigley, Joseph E., Mischke, Charles R., Budynas, Richard G., 2003, Mechanical engineering design.

[4] Callister, William D., 2003, Materials science and engineering: an introduction, New York: John Wiley $\&$ Sons.

[5] www.HoneybeeRobotics.com

[6] www.spacedaily.com/news/mars-driller-00a1.html

[7] www.space.com/scienceastronomy/solarsystem/

mars_drilling_000623.html

[8] www.jpl.nasa.gov/missions/mer/daily.cfm?date $=3$

[9] www.nasa.gov

[10] www.hagby.se/english/miningwire.html

[11] www.jpl.nasa.gov/releases/2002/

release_2002_6.html

[12] Jones, Howard, The ESA MICRO ROSA2

Activity, Conclusion and Future plans report.

[13] Jones, Howard, The ASI "DeeDri" drill tool prototypes and drilling system developments for Mars soil sampling applications, Report.

[14] www.store.yahoo.com/drillcity

[15] www.drillbitcity.com/

[16] http://www.windows.ucar.edu/tour/link=/mars/

lower_atmosphere.html 